\title{
One More Step Towards Well-Composedness of Cell Complexes over nD Pictures
}

\author{
Nicolas Boutry ${ }^{1}$, Rocio Gonzalez-Diaz ${ }^{2}$, and Maria-Jose Jimenez ${ }^{2(凶)}$ \\ 1 EPITA Research and Development Laboratory (LRDE), \\ Le Kremlin-Bicêtre, France \\ nicolas.boutry@lrde.epita.efr \\ 2 Departamento Matematica Aplicada I, Universidad de Sevilla, \\ Campus Reina Mercedes, 41012 Sevilla, Spain \\ \{rogodi,majiro\}@us.es
}

\begin{abstract}
An nD pure regular cell complex $K$ is weakly well-composed (wWC) if, for each vertex $v$ of $K$, the set of $n$-cells incident to $v$ is face-connected. In previous work we proved that if an $\mathrm{nD}$ picture $I$ is digitally well composed (DWC) then the cubical complex $Q(I)$ associated to $I$ is wWC. If $I$ is not DWC, we proposed a combinatorial algorithm to "locally repair" $Q(I)$ obtaining an $\mathrm{nD}$ pure simplicial complex $P_{S}(I)$ homotopy equivalent to $Q(I)$ which is always wWC. In this paper we give a combinatorial procedure to compute a simplicial complex $P_{S}(\bar{I})$ which decomposes the complement space of $\left|P_{S}(I)\right|$ and prove that $P_{S}(\bar{I})$ is also wWC. This paper means one more step on the way to our ultimate goal: to prove that the $\mathrm{nD}$ repaired complex is continuously well-composed (CWC), that is, the boundary of its continuous analog is an $(n-1)$ manifold.
\end{abstract}

\section{Introduction}

Ensuring that the cellular decomposition $K$ of an object is continuously wellcomposed (CWC) allows to later work in a more efficient way. For example in the 3-dimensional (3D) setting, it allows to work with surface parameterizations [9]. Besides, $K$ being CWC has also topological computation benefits in the sense that the homology of the object can be deduced from the homology of its boundary $[7,8]$. Moreover, if the boundary of the object is not a manifold, then it is well-known that the multigrid convergence of some geometrical estimators is slower (see, for example, [14]).

The authors have been working for several years in providing algorithms to "locally repair" the cubical complex $Q(I)$ canonically associated to a given picture $I$, in order to obtain a cell complex homotopic to $Q(I)$ and CWC.

A first attempt to solve the problem in the 3-dimensional setting (3D) was presented in [10]. In [11-13], the authors provided a satisfactory solution in the $3 \mathrm{D}$ case to locally repair $Q(I)$ following a purely combinatorial procedure, obtaining a polyhedral $P(I)$ that is CWC.

Authors' names listed in alphabetical order.

(C) Springer Nature Switzerland AG 2019

M. Couprie et al. (Eds.): DGCI 2019, LNCS 11414, pp. 101-114, 2019.

https://doi.org/10.1007/978-3-030-14085-4_9 


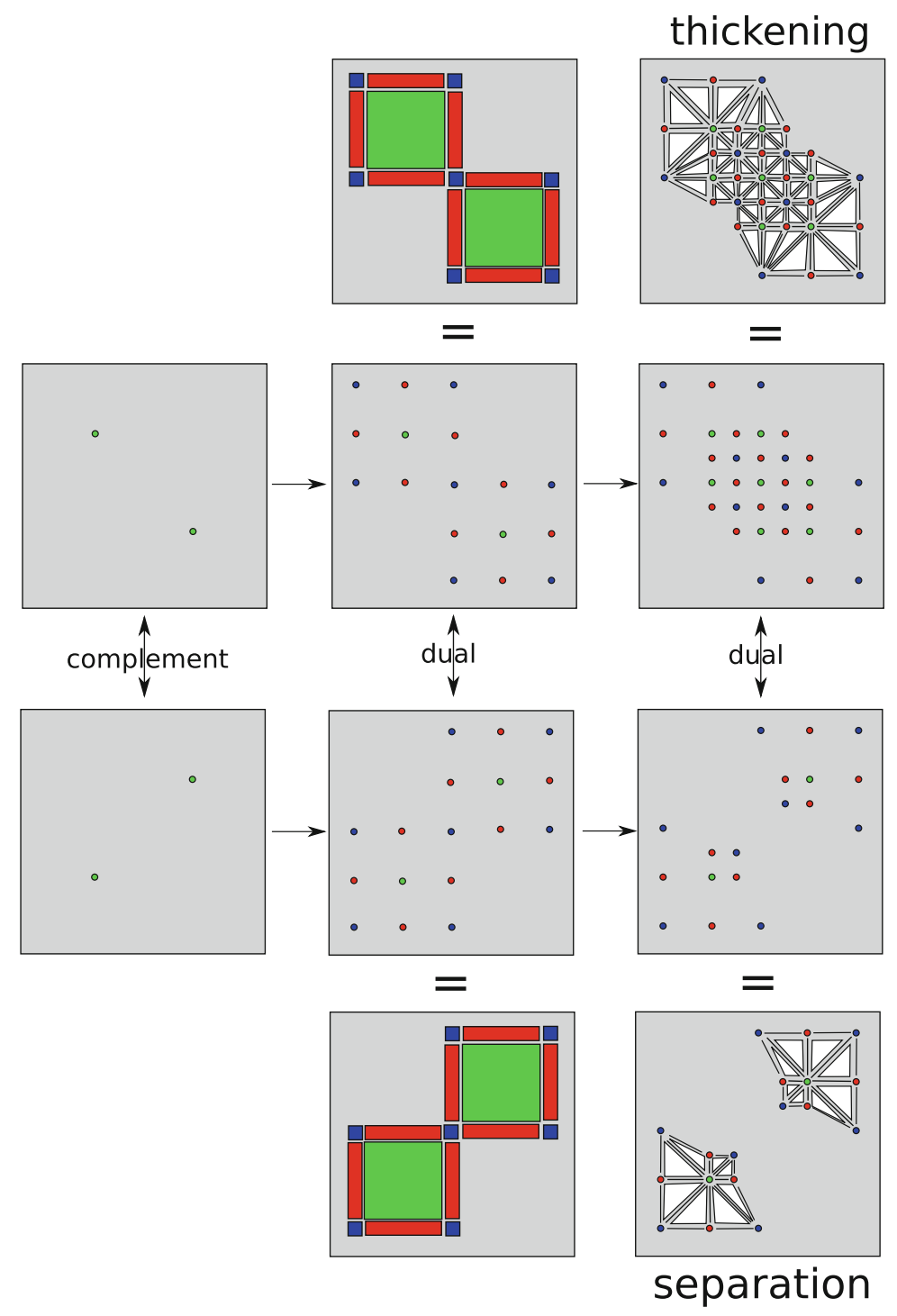

Fig. 1. $P_{S}(I)$ versus $P_{S}(\bar{I})$. In the upper half, starting from an image $I$ made of two (green) points (on the left), we compute its associated cubical complex $Q(I)$ (in the middle column). When a critical configuration is detected in $I$, a reparation procedure is applied on $Q(I)$, leading to a well-composed simplicial complex $P_{S}(I)$ (on the right). In the lower half, we compute the complement $\bar{I}$ of the image $I$ which leads to the dual cubical complex $Q(\bar{I})$ (in the middle). Now, the reparation procedure (which is described in this paper) "separates" the complex $Q(\bar{I})$ to yield a new simplicial complex $P_{S}(\bar{I})$ (on the right), which is also weakly well-composed. Notice that the picture of $\bar{I}$ (and hence, $Q(\bar{I})$ and $\left.P_{S}(\bar{I})\right)$ has been restricted to a two-by-two pixels context and the infinite set of pixels around them are missed. (Color figure online) 
In [3], we extend to any dimension $(\mathrm{nD})$ the previous method: Given an $\mathrm{nD}$ picture $I$, we detect non-well-composed configurations (which cause $Q(I)$ not to be CWC) and apply on those configurations a repairing process (see the upside part of Fig. 1 for a sketch of the procedure) consisting in "thickening" the picture representation with some new cells in order to remove the "pinches". The resulting computed complex $P_{S}(I)$ is a simplicial complex homotopic to $Q(I)$. In that paper, as a first step towards continuous well-composedness, we proved that $P_{S}(I)$ is weakly well-composed (wWC), what means that there always exists a face-connected path in $P_{S}(I)$ of $n$-simplices incident to a common vertex $v$, joining any two $n$-simplices incident to $v$. Some other recent works following the idea of the reparation method are [1] (using the inter-simplex Chebyshev distance), [5] (for face centered cubic grids) and [6] (for body centered cubic grids). A tutorial revisiting the different concepts on well-composedness depending on the nature of the object of study is [2].

Proving that $P_{S}(I)$ is CWC in any dimension is not an easy task and is still an open problem. CWCness is self-dual, that is, the boundary of a compact subset $X$ of $\mathbb{R}^{n}$ is a manifold iff the boundary of its complement $X^{c}:=\mathbb{R}^{n} \backslash X$ is a manifold too. Hence, proving that $P_{S}(I)$ and its dual $P_{S}(\bar{I})$, where $\bar{I}$ is the complement of $I$ (see the lower half of Fig. 1), are both wWC, shows that the type of wellcomposedness of $P_{S}(I)$ we are proving here is also self-dual and then stronger than simply weak well-composedness. Consequently, in this paper we go one step towards the final goal, by proving that $P_{S}(\bar{I})$ is also wWC. The paper is organized as follows: Sect. 2 is devoted to recall the main concepts needed to understand the rest of the paper and we sketch the method developed in [3] to compute $P_{S}(I)$. In Sect. 3 we introduce the complement of an $\mathrm{nD}$ picture denoted by $\bar{I}$ and its canonically associated cubical complex $Q(\bar{I})$. In Sect. 4, we explain the procedure to locally repair $Q(\bar{I})$ to obtain the wWC simplicial complex $P_{S}(\bar{I})$, satisfying that its continuous analog is the closure of the complement space of $P_{S}(I)$. This procedure is a modification of the one given in [3] to compute $P_{S}(I)$. Section 5 is devoted to conclusions and future works.

\section{Background}

Let $n \geq 2$ be an integer and $\mathbb{Z}^{n}$ the set of points with integer coordinates in $\mathrm{nD}$ space $\mathbb{R}^{n}$. An $n D$ binary image is a pair $I=\left(\mathbb{Z}^{n}, F_{I}\right)$ where $F_{I}$ is a finite subset of $\mathbb{Z}^{n}$ called foreground of $I$. If $I=\left(\mathbb{Z}^{n}, F_{I}\right)$, with $F_{I} \subset 4 \mathbb{Z}^{n}$, then we say that $I$ is an $n D$ picture. This is only a technical issue: we can encode the cubical complex associated to $I$ in $2 \mathbb{Z}^{n}$, but we need the foreground $F_{I}$ to be included in $4 \mathbb{Z}^{n}$ so that we have "extra" space to encode the repairing cells that will be added to "thicken" the non-manifold parts of such cubical complex.

For two integers $k \leq k^{\prime}$, let $\llbracket k, k^{\prime} \rrbracket$ denote the set $\left\{k, k+1, \ldots, k^{\prime}-1, k^{\prime}\right\}$ and $\mathbb{B}=\left\{e^{1}, \ldots, e^{n}\right\}$ the canonical basis of $\mathbb{Z}^{n}$. 
Given a point $z \in 4 \mathbb{Z}^{n}$ and a family of vectors $\mathcal{F}=\left\{f^{1}, \ldots, f^{k}\right\} \subseteq \mathbb{B}$, the block of dimension $k$ associated to the couple $(z, \mathcal{F})$ is the set defined as:

$$
B(z, \mathcal{F})=\left\{z+\sum_{i \in \llbracket 1, k \rrbracket} \lambda_{i} f^{i}: \lambda_{i} \in\{0,4\}, \forall i \in \llbracket 1, k \rrbracket\right\} .
$$

This way, a 0-block is a point, a 1-block is an edge, a 2-block is a square, and so on. A subset $B \subset 4 \mathbb{Z}^{n}$ is called a block if there exists a couple $(z, \mathcal{F}) \in$ $4 \mathbb{Z}^{n} \times \mathcal{P}(\mathbb{B})^{1}$ such that $B=B(z, \mathcal{F})$. We will denote the set of blocks of $4 \mathbb{Z}^{n}$ by $\mathcal{B}\left(4 \mathbb{Z}^{n}\right)$.

Two points $p, q$ belonging to a block $B \in \mathcal{B}\left(4 \mathbb{Z}^{n}\right)$ are said to be antagonists in $B$ if their distance equals the maximum distance using the $L^{1}$-norm ${ }^{2}$ between two points in $B$ :

$$
\|p-q\|_{1}=\max \left\{\|r-s\|_{1} ; r, s \in B\right\} .
$$

The antagonist of a point $p$ in a block $B \in \mathcal{B}\left(4 \mathbb{Z}^{n}\right)$ containing $p$ exists and is unique. It is denoted by $\operatorname{antag}_{B}(p)$. Note that when two points $\left(x_{1}, \ldots, x_{n}\right)$ and $\left(y_{1}, \ldots, y_{n}\right)$ are antagonists in a block of dimension $k \in \llbracket 0, n \rrbracket$, then $\left|x_{i}-y_{i}\right|=4$ for $i \in\left\{i_{1}, \ldots, i_{k}\right\} \subseteq \llbracket 1, n \rrbracket$ and $x_{i}=y_{i}$ otherwise.

Now, let $I=\left(\mathbb{Z}^{n}, F_{I}\right)$ be an $\mathrm{nD}$ picture and $B \in \mathcal{B}\left(4 \mathbb{Z}^{n}\right)$ a block of dimension $k \in \llbracket 2, n \rrbracket$. We say that $I$ contains a critical configuration $(\mathrm{CC})$ in the block $B$ if $F_{I} \cap B=\left\{p, p^{\prime}\right\}$ or $F_{I} \cap B=B \backslash\left\{p, p^{\prime}\right\}$, with $p, p^{\prime}$ being two antagonists in $B$.

DWCness. The $\mathrm{nD}$ picture $I=\left(\mathbb{Z}^{n}, F_{I}\right)$ is said to be digitally well-composed (DWC) if for any block $B$ of dimension $k \in \llbracket 2, n \rrbracket$, the set $F_{I} \cap B$ is not a critical configuration. Additionally, $\bar{I}=\left(\mathbb{Z}^{n}, 4 \mathbb{Z}^{n} \backslash F_{I}\right)$ is DWC iff $I$ is DWC (we call this property self-duality [4] of DWCness).

We say that two elements $x, y$ of $4 \mathbb{Z}^{n}$ are $2 n$-neighbors in $4 \mathbb{Z}^{n}$ when there exists some $i \in \llbracket 1, n \rrbracket$ verifying that $x_{i}=y_{i} \pm 4$ and for any $j \in \llbracket 1, n \rrbracket \backslash\{i\}, x_{j}=$ $y_{j}$. Also, we call $2 n$-path joining $p, q$ in $S \subseteq 4 \mathbb{Z}^{n}$ a sequence $\left(p^{0}=p, p^{1}, \ldots, p^{k}=\right.$ $q$ ) of elements of $S$ which verifies for any $i \in \llbracket 0, k-1 \rrbracket$ that $p^{i}$ and $p^{i+1}$ are $2 n$-neighbours in $4 \mathbb{Z}^{n}$.

Proposition 1 ([3]). If $I=\left(\mathbb{Z}^{n}, F_{I}\right)$ is $D W C$ then, for any block $B \in \mathcal{B}\left(4 \mathbb{Z}^{n}\right)$ and for any two points $p, q$ in $F_{I} \cap B$, there exists a $2 n$-path in $F_{I} \cap B$ joining $p$ and $q$.

We use the term $n D$ cell complex $K$ to refer to a pure regular cell complex of rank $n$ embedded in $\mathbb{R}^{n}$. The underlying space (i.e., the union of the $n$-cells as subspaces of $\mathbb{R}^{n}$ ) will be denoted by $|K|$. Regular cell complexes have particularly nice properties, for example, their homology is effectively computable (see $[15$, p. 243]).

\footnotetext{
${ }^{1}$ The expression $\mathcal{P}(\mathbb{B})$ represents the set of all the subsets of $\mathbb{B}$.

2 The $L^{1}$-norm of a vector $\alpha=\left(x_{1}, \ldots, x_{n}\right)$ is $\|\alpha\|_{1}=\sum_{i \in \llbracket 1, n \rrbracket}\left|x_{i}\right|$.
} 
The boundary surface $\partial K$ of an $\mathrm{nD}$ cell complex $K$ is the (n-1)D cell complex composed by the $(n-1)$-cells of $K$ that are faces of exactly one $n$-cell, together with all their faces.

The cone (join) on a simplicial complex $K$ with vertex $v$, denoted by $v * K$ is the simplicial complex whose simplices have the form $\left\langle v_{0}, \ldots, v_{\ell}, v\right\rangle$ (where $\left\langle v_{0}, \ldots, v_{\ell}\right\rangle$ is a simplex of $K$ spanned by the set of points $\left.\left\{v_{0}, \ldots, v_{\ell}\right\}\right)$, along with all the faces of such simplices.

CWCness. An $\mathrm{nD}$ cell complex $K$ is said to be continuously well-composed $(\mathrm{CWC})$ if $|\partial K|$ is an $(n-1)$-manifold, that is, each point of $|\partial K|$ has a neighborhood homeomorphic to $\mathbb{R}^{n-1}$ into $|\partial K|$.

Let $\mathcal{S}$ be a set of $\ell$-cells of $K, \ell \in \llbracket 1, n \rrbracket$. We say that two $\ell$-cells $\sigma$ and $\sigma^{\prime}$ are face-connected in $\mathcal{S}$ if there exists a path $\pi\left(\sigma, \sigma^{\prime}\right)=\left(\sigma_{1}=\sigma, \sigma_{2} \ldots, \sigma_{m-1}, \sigma_{m}=\right.$ $\left.\sigma^{\prime}\right)$ of $\ell$-cells of $\mathcal{S}$ such that for any $i \in \llbracket 1, m-1 \rrbracket, \sigma_{i}$ and $\sigma_{i+1}$ share exactly one $(\ell-1)$-cell of $K$. The set $\mathcal{S}$ is face-connected if any two $\ell$-cells $\sigma$ and $\sigma^{\prime}$ in $\mathcal{S}$ are face-connected in $\mathcal{S}$.

wWCness. An $\mathrm{nD}$ cell complex $K$ is weakly well-composed ( $w W C$ ) if for any 0cell $\mu$ in $K$, the set of $n$-cells incident to $\mu$, denoted by $\mathcal{A}_{K}^{(n)}(\mu)$, is face-connected.

A canonical size- $4 n$-cube is a size- $4 n$-cube centered at a point in $\mathbb{Z}^{n}$ having their $(n-1)$-faces parallel to the coordinate hyperplanes.

Given an $\mathrm{nD}$ picture $I$, the $\mathrm{nD}$ cell complex whose $n$-cells are the canonical size- $4 n$-cubes centered at each point in $F_{I}$ is denoted by $Q(I)$.

We say that $J=\left(\mathbb{Z}^{n}, F_{J}\right)$ encodes $Q(I)$ if $F_{J}$ is the set of barycenters of the cells in $Q(I)^{3}$. We say that $p \in F_{J}$ encodes $\sigma \in Q(I)$ if $p$ is the barycenter of $\sigma$. In that case, we denote $\sigma$ as $\sigma_{Q(I)}(p)$.

Notation 1. Let $N, M \in \mathbb{Z}$ such that $0 \leq N<M$. Let $p=\left(x_{1}, \ldots, x_{n}\right) \in \mathbb{Z}^{n}$. Then $N_{M}(p)$ denotes the set of indices $\left\{i \in \llbracket 1, n \rrbracket: x_{i} \equiv N \bmod M\right\}$.

Remark 1. $2 \mathbb{Z}^{n}$ can be decomposed into the disjoint sets

$$
\mathcal{E}_{\ell}:=\left\{p \in 2 \mathbb{Z}^{n}: \operatorname{card}\left(0_{4}(p)\right) \text { is } \ell\right\}
$$

where $\operatorname{card}(S)$ is the number of elements in the set $S$. For example $\mathcal{E}_{n}=4 \mathbb{Z}^{n}$ and $\mathcal{E}_{0}=(2 \mathbb{Z} \backslash 4 \mathbb{Z})^{n}$. Besides, $\mathbb{Z}^{n} \backslash 2 \mathbb{Z}^{n}$ can be decomposed into the disjoint sets:

$$
\mathcal{O}_{\ell}:=\left\{p \in \mathbb{Z}^{n} \backslash 2 \mathbb{Z}^{n}: \operatorname{card}\left(0_{2}(p)\right) \text { is } \ell\right\} .
$$

Notice that $\ell$-cells of the cubical complex $Q(I)$ are encoded by points in $\mathcal{E}_{\ell}$. Intuitively, sets $\mathcal{O}_{\ell}$ will serve as support to build new cells, in the repairing process, around critical configurations.

Proposition 2 ([3]). The set of points of $F_{J}$ encoding the faces of a cell $\sigma_{Q(I)}(p)$ is:

$\mathcal{D}_{F_{J}}(p):=\mathcal{D}_{F_{J}}^{+}(p) \backslash\{p\} \quad$ where $\mathcal{D}_{F_{J}}^{+}(p)=\left\{p+\sum_{j \in 0_{4}(p)} \lambda_{j} e^{j}: \lambda_{j} \in\{0, \pm 2\}\right\}$.

\footnotetext{
${ }^{3}$ Observe that $F_{J} \subset 2 \mathbb{Z}^{n}$.
} 
The set of points encoding the $i$-faces of $\sigma_{Q(I)}(p)$ will be denoted by $\mathcal{D}_{F_{J}}^{i}(p)$ and is, in fact, $\mathcal{D}_{F_{J}}(p) \cap \mathcal{E}_{i}$. In particular, the set of points encoding the vertices of $\sigma_{Q(I)}(p)$ is

$$
\mathcal{D}_{F_{J}}^{0}(p)=\left\{p+\sum_{j \in 0_{4}(p)} \lambda_{j} e^{j}: \lambda_{j} \in\{ \pm 2\}\right\}
$$

Proposition 3 ([3]). The set of points of $F_{J}$ encoding the cells incident to a cell $\sigma_{Q(I)}(p)$ is:

$\mathcal{A}_{F_{J}}(p):=\mathcal{A}_{F_{J}}^{+}(p) \backslash\{p\}$ where $\mathcal{A}_{F_{J}}^{+}(p)=\left\{p+\sum_{j \in 2_{4}(p)} \lambda_{j} e^{j}: \lambda_{j} \in\{0, \pm 2\}\right\} \cap F_{J}$.

The set of points encoding the $i$-cells incident to $\sigma_{Q(I)}(p)$ will be denoted by $\mathcal{A}_{F_{J}}^{i}(p)$. In particular,

$$
\mathcal{A}_{F_{J}}^{n}(p):=F_{J} \cap\left\{p+\sum_{j \in 2_{4}(p)} \lambda_{j} e^{j}: \lambda_{j} \in\{ \pm 2\}\right\} .
$$

Proposition 4 ([3]). If $I=\left(\mathbb{Z}^{n}, F_{I}\right)$ is $D W C$, then $Q(I)$ is $w W C$.

Cell Complexes over nD Pictures. A cell complex over an nD picture I is an $\mathrm{nD}$ cell complex, denoted by $K(I)$, such that there exists a deformation retraction from $K(I)$ onto $Q(I)$.

In [3] we detailed a procedure to "locally repair" the cubical complex $Q(I)$ when $I=\left(\mathbb{Z}^{n}, F_{I}\right)$ is not DWC to obtain a simplicial complex $P_{S}(I)$ such that $\left|P_{S}(I)\right|$ is homotopy equivalent to $|Q(I)|$ and $P_{S}(I)$ is a wWC simplicial complex. The principal steps are summarized below:

First, Procedure 1 from [3] is used to compute the set $R$ of (critical) points of $F_{J}$ representing the critical cells in $Q(I)$. Basically, we look for critical configurations and for the cells incident to vertices involved in such critical configurations. These cells are the candidates to be repaired in order to obtain the wWC simplicial complex $P_{S}(I)$. We reproduce below the procedure so that the paper is self-contained.

Once the critical points are computed, extra points are added around them, defining the set $F_{L}$ that will contain all the vertices of the new construction $P_{S}(I)$ :

$$
F_{L}:=F_{J} \cup \bigcup_{p \in R} S(p)
$$

where $S(p):=\left\{p+\sum_{j \in 2_{4}(p)} \lambda_{j} e^{j}: \lambda_{j} \in\{0, \pm 1\}\right\}$. These points are classified into sets $\mathcal{C}_{\ell}$, for $\ell \in \llbracket 0, n \rrbracket$, defined as follows:

$$
\mathcal{C}_{n}:=\left(\mathcal{E}_{n} \cap F_{L}\right) \cup R \text { and } \mathcal{C}_{\ell}:=\left(\left(\mathcal{E}_{\ell} \backslash R\right) \cup \mathcal{O}_{\ell}\right) \cap F_{L} \text { for } \ell \in \llbracket 0, n-1 \rrbracket,
$$

where $\mathcal{E}_{\ell}$ and $\mathcal{O}_{\ell}$ were defined in Remark 1 . 


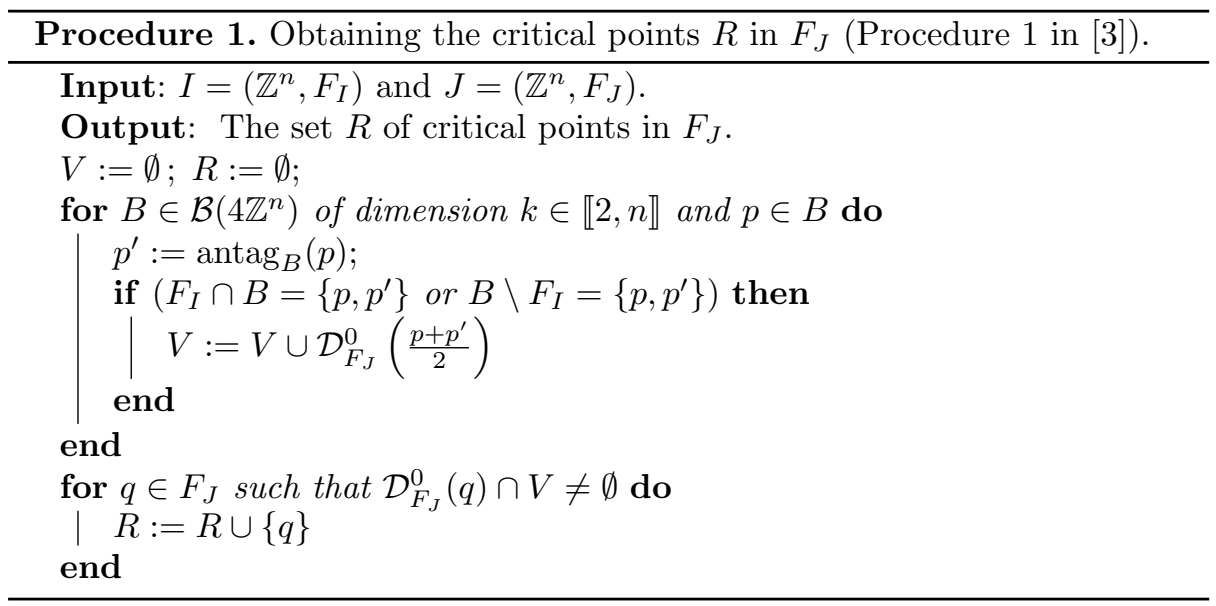

Finally, $F_{L}$ is the set of vertices used to compute $P_{S}(I)$ successively applying the cone join operation $(*)$. For this construction, new sets $\mathcal{D}_{F_{L}}(p)$, for each $p \in F_{L}$, are defined.

Definition 1 ([3]). For $p \in F_{L}$, define the set $\mathcal{D}_{F_{L}}(p):=\mathcal{D}_{F_{L}}^{+}(p) \backslash\{p\}$ where:

- If $p \in \mathcal{C}_{0}$ then $\mathcal{D}_{F_{L}}^{+}(p):=\{p\}$

- If $p \in \mathcal{E}_{\ell} \backslash R$, for $\ell \in \llbracket 1, n \rrbracket$, then $\mathcal{D}_{F_{L}}^{+}(p):=\mathcal{D}_{F_{J}}^{+}(p)$;

- If $p \in \mathcal{E}_{\ell} \cap R$, for $\ell \in \llbracket 1, n \rrbracket$, then

$$
\mathcal{D}_{F_{L}}^{+}(p):=S(p) \sqcup\left(\mathcal{D}_{F_{J}}(p) \backslash R\right) \sqcup \bigsqcup_{r \in \mathcal{D}_{F_{J}}(p) \cap R}(S(r) \cap \mathcal{N}(p)) ;
$$

- If $p \in \mathcal{O}_{\ell}$, for $\ell \in \llbracket 1, n-1 \rrbracket$, then $\exists ! q \in R$ s.t. $p \in S(q)$. We have:

$$
\mathcal{D}_{F_{L}}^{+}(p):=\left(S(q) \cap \mathcal{N}^{+}(p)\right) \sqcup\left(\mathcal{D}_{F_{J}}(q) \backslash R\right) \sqcup \bigsqcup_{r \in \mathcal{D}_{F_{J}}(q) \cap R}(S(r) \cap \mathcal{N}(p)) ;
$$

Where $\mathcal{N}^{+}(p):=\left\{p+\sum_{j \in 0_{2}(p)} \lambda_{j} e^{j}: \lambda_{j} \in\{0, \pm 1\}\right\}$ and $\mathcal{N}(p):=\mathcal{N}^{+}(p) \backslash\{p\}$.

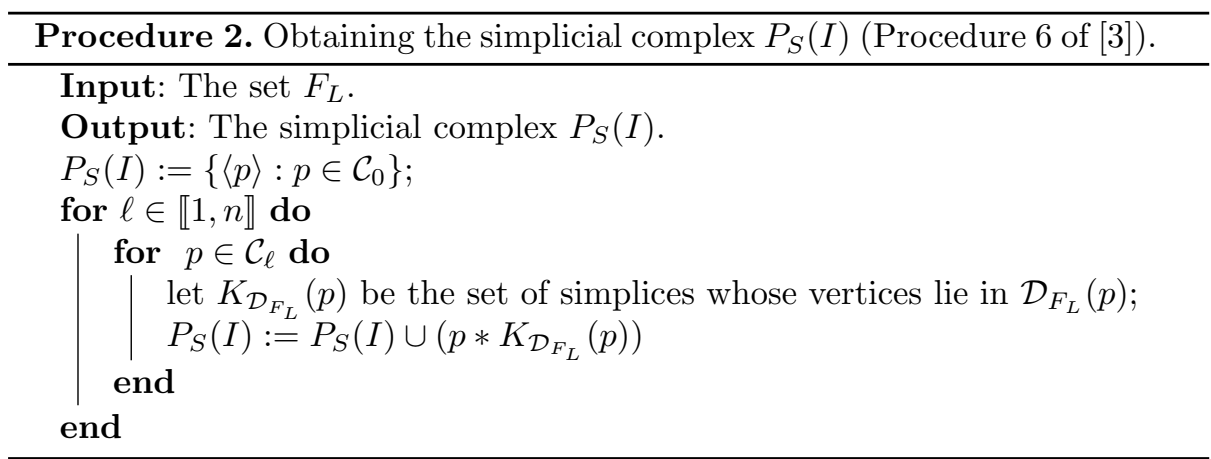

Proposition 5 ([3]). The simplicial complex $P_{S}(I)$ is always $w W C$. 


\section{The Complement of an nD Digital Picture $I$}

The complement of an $\mathrm{nD}$ picture $I=\left(\mathbb{Z}^{n}, F_{I}\right)$ is the pair $\bar{I}=\left(\mathbb{Z}^{n}, 4 \mathbb{Z}^{n} \backslash F_{I}\right)$. Observe that $\bar{I}$ is not an $\mathrm{nD}$ picture (as defined in this paper) since $4 \mathbb{Z}^{n} \backslash F_{I}$ is not finite.

The $\mathrm{nD}$ cell complex whose $n$-cells are the canonical size- $4 n$-cubes centered at each point in $4 \mathbb{Z}^{n} \backslash F_{I}$ is denoted by $Q(\bar{I})$.

DWCness is self-dual in the sense that an $\mathrm{nD}$ picture $I=\left(\mathbb{Z}^{n}, F_{I}\right)$ is DWC iff its complement $\bar{I}=\left(\mathbb{Z}^{n}, 4 \mathbb{Z}^{n} \backslash F_{I}\right)$ is DWC. The following proposition is a property of DWCness (see Theorem 1 in [4]) adapted to $\mathrm{nD}$ pictures.

Proposition 6 ([4]). When $I=\left(\mathbb{Z}^{n}, F_{I}\right)$ is a $D W C n D$ picture, then for any block $B \in \mathcal{B}\left(4 \mathbb{Z}^{n}\right)$, and for any $p, q \in B \backslash F_{I}$ which are antagonists in $B$, there exists a $2 n$-path joining $p$ and $q$ in $B \backslash F_{I}$.

Then we obtain the following result.

Proposition 7. If $I=\left(\mathbb{Z}^{n}, F_{I}\right)$ is $D W C$ then, for any block $B \in \mathcal{B}\left(4 \mathbb{Z}^{n}\right)$ and for any two points $p, q$ in $B \backslash F_{I}$, there exists a $2 n$-path in $B \backslash F_{I}$ from $p$ to $q$.

Proof. Similarly to the proof of Proposition 5 in [3], let $B \in \mathcal{B}\left(4 \mathbb{Z}^{n}\right)$ be a block such that $B \backslash F_{I}$ has at least two points. For any two points $p, q \in B \backslash F_{I}$, there exists a block $B^{\prime} \subseteq B$ such that $q=\operatorname{antag}_{B^{\prime}}(p)$. Then by Proposition 6 , there exists a $2 n$-path joining $p$ and $q$ in $B^{\prime} \backslash F_{I} \subseteq B \backslash F_{I}$.

Proposition 8. Let $J^{\prime}=\left(\mathbb{Z}^{n}, F_{J}^{\prime}\right)$ encoding $Q(\bar{I})$. Then:

$-F_{J} \cup F_{J}^{\prime}=2 \mathbb{Z}^{n}$

- $\left(\mathbb{Z}^{n}, F_{J} \cap F_{J}^{\prime}\right)$ encodes $\partial Q(I)$.

Proof. First, by construction, $F_{J} \cup F_{J}^{\prime} \subset 2 \mathbb{Z}^{n}$. Let us assume that a point $p \in$ $2 \mathbb{Z}^{n}$ belongs to $\mathcal{E}_{\ell}$. Consider $p^{\prime}=p+\sum_{j \in 2_{4}(p)} 2 e^{j}$. Then, either $p^{\prime} \in F_{I}$ or $p^{\prime} \in 4 \mathbb{Z}^{n} \backslash F_{I}$, and hence, $p$ encodes either, an $\ell$-face of an $n$-cube of $Q(I)$ or an $\ell$-face of an $n$-cube of $Q(\bar{I})$. That is, $p \in F_{J} \cup F_{J}^{\prime}$.

Let $p=\left(x_{1}, \ldots, x_{n}\right) \in \mathcal{E}_{n-1}$ such that $p$ encodes an $(n-1)$-cell of $\partial Q(I)$. Then, without loss of generality, we can assume that $x_{i} \equiv 0 \bmod 4$ for $i \in$ $\llbracket 1, n-1 \rrbracket$ and $x_{n} \equiv 2 \bmod 4$. Consider the two points in $4 \mathbb{Z}^{n} q=p+2 e^{n}$ and $q^{\prime}=p-2 e^{n}$. Necessarily, one of them lies in $F_{I}$ and the other one in $4 \mathbb{Z}^{n} \backslash F_{I}$, so $p \in F_{J} \cap F_{J}^{\prime}$. If $p$ encodes an $\ell$-cell of $\partial Q(I)$, then, $p$ is a face of an $(n-1)$ cell of $\partial Q(I)$ and the previous argument applies to conclude that $p \in F_{J} \cap F_{J}^{\prime}$. Conversely, if $p \in F_{J} \cap F_{J}^{\prime}$ (assume that $p \in \mathcal{E}_{\ell}$ ), then $p$ encodes an $\ell$-face of an $n$-1-cell in $Q(I)$ which is a face of both an $n$-cube in $Q(I)$ and an $n$-cube in $Q(\bar{I})$ (otherwise, it could not happen that $p \in F_{J} \cap F_{J}^{\prime}$ ). Then, $p$ encodes a cell in $\partial Q(I)$. Notice that the same argument proves that $p$ encodes a cell in $\partial Q(\bar{I})$.

Proposition 9. If $I$ is $D W C$ then $Q(\bar{I})$ is $w W C$.

Proof. When $I$ is DWC, $\bar{I}$ is DWC by definition of DWCness. Then $Q(\bar{I})$ is wWC by Proposition 4. 


\section{The Simplicial Complex $P_{S}(\bar{I})$}

Similar to the work made in [3], we now detail a procedure to "locally repair" the cubical complex $Q(\bar{I})$ when $I=\left(\mathbb{Z}^{n}, F_{I}\right)$ is not DWC obtaining a simplicial complex $P_{S}(\bar{I})$ which is always wWC.

First, Procedure 1 is used to compute the set of points $R^{\prime}$ representing the critical cells in $Q(\bar{I})$, considering as input $I=\left(\mathbb{Z}^{n}, F_{I}\right)$ and $J^{\prime}=\left(\mathbb{Z}^{n}, F_{J}^{\prime}\right)$.

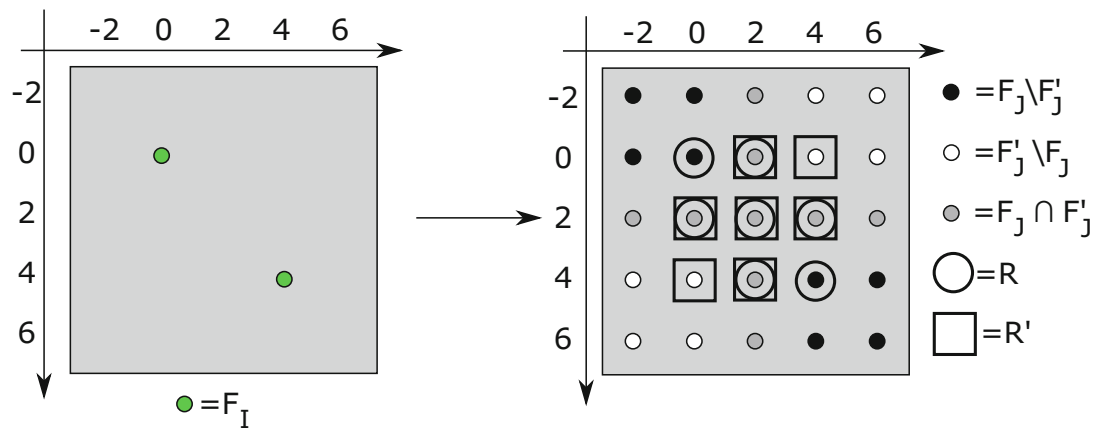

Fig. 2. The difference between $R$ and $R^{\prime}$.

Remark 2. In general, $R \cap R^{\prime} \neq \emptyset, R, R^{\prime}$ (see Fig. 2).

Second, once the critical points $R^{\prime}$ are computed, the points of $F_{J}^{\prime}$ which do not belong to $R^{\prime}$ are preserved, the points of $R^{\prime}$ are replaced by a set of points using an operator $S^{\prime}($.) introduced below. This part of the process is the one that differs from that in [3].

- If $p \in R^{\prime} \backslash R$ (that is, $\sigma_{Q(\bar{I})}(p)$ is not in $\left.\partial Q(I)\right)$ then $S^{\prime}(p):=S(p)$.

- If $p \in R^{\prime} \cap R$ (that is, $\sigma_{Q(\bar{I})}(p)$ is in $\left.\partial Q(I)\right)$ then

$$
S^{\prime}(p):=\left\{p+\sum_{j \in 2_{4}(p)} \lambda_{j} e^{j}: \lambda_{j} \in\{0, \pm 1\} \text { and } p+\sum_{j \in 2_{4}(p)} 2 \lambda_{j} e^{j} \in R^{\prime} \backslash R\right\} .
$$

Figure 3 shows small examples of sets $S^{\prime}(p)$. Then, the set $F_{L}^{\prime}$ is defined as:

$$
F_{L}^{\prime}:=\left(F_{J}^{\prime} \backslash R^{\prime}\right) \cup \bigcup_{p \in R^{\prime}} S^{\prime}(p)
$$

Observe that not all the points in $F_{J}^{\prime}$ belong to $F_{L}^{\prime}$, contrary to what happened with $F_{L}$. 

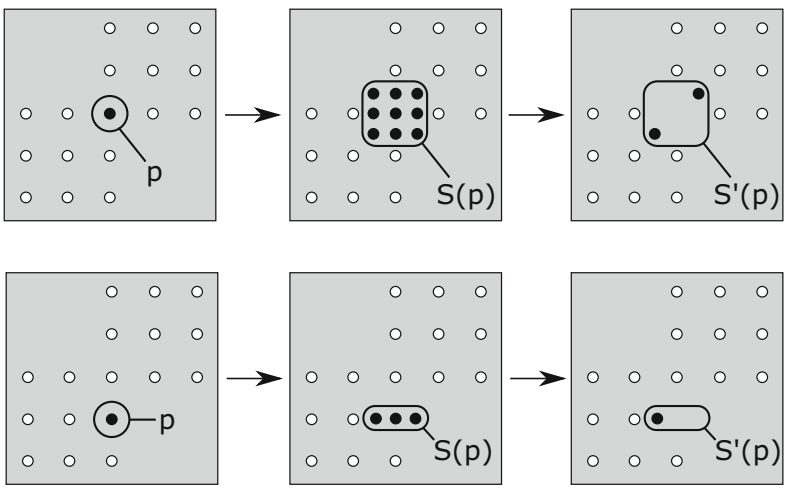

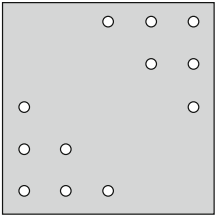

$\mathrm{F}_{\mathrm{J}}^{\prime} \backslash \mathrm{R}$

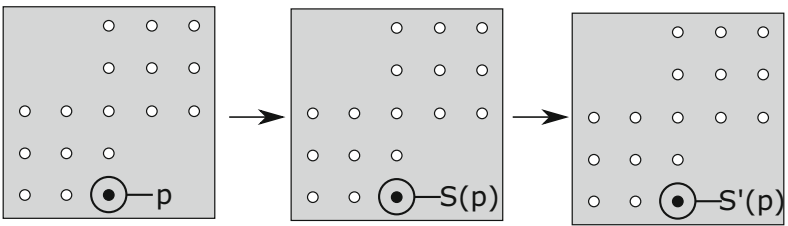

Fig. 3. Computation of $S^{\prime}(p)$. From $F_{J}^{\prime}$ depicted in the upper left part of the figure by the black and white points, we deduce $S(p)$, and then $S^{\prime}(p)$ which is the subset of $S(p)$ satisfying that they can be written $p+\sum_{j \in 2_{4}(p)} 2 \lambda_{j} e^{j} \in F_{J}^{\prime} \backslash R$, with $\lambda_{j} \in\{0, \pm 1\}$.

Remark 3. As it happened with $F_{L}$ in [3], the set $F_{L}^{\prime}$ can be decomposed into the disjoint sets:

$$
\begin{gathered}
\mathcal{C}_{n}^{\prime}:=\left(\mathcal{E}_{n} \cap F_{L}^{\prime}\right) \cup\left(R^{\prime} \backslash R\right) \\
\mathcal{C}_{\ell}^{\prime}:=\left(\left(\mathcal{E}_{\ell} \backslash R^{\prime}\right) \cup \mathcal{O}_{\ell}\right) \cap F_{L}^{\prime} \text { for } \ell \in \llbracket 0, n-1 \rrbracket .
\end{gathered}
$$

Third, to compute $P_{S}(\bar{I})$, we cannot directly use Procedure 6 of $[3]$ since $F_{L}^{\prime}$ has an infinite number of points. Now, the idea is to compute $P_{S}(\bar{I})$ locally around points in $\mathcal{C}_{n}^{\prime}$ :

- For each $p$ in $\left(\mathcal{E}_{n} \cap F_{L}^{\prime}\right) \backslash R^{\prime}$, apply Procedure 2 with $\mathcal{D}_{F_{L}^{\prime}}^{+}(p)^{4}$ as input. This process will produce a simplicial subdivision of the canonical 4 -size $n$-cube.

- For the whole set $R^{\prime} \backslash R$, apply Procedure 2 with

$$
\bigcup_{p \in R^{\prime} \backslash R} \mathcal{D}_{F_{L}^{\prime}}^{+}(p)
$$

as input (a finite set), what will produce the rest of the simplices in $P_{S}(\bar{I})$.

A complete procedure used to compute the simplicial complex $P_{S}(\bar{I})$ over an nD picture $I$ which consists in two pixels sharing a vertex, is showed in Fig. 4.

${ }^{4}$ Definition of $\mathcal{D}_{F_{L}^{\prime}}^{+}(p)$ can be obtained from Definition 1 replacing $F_{L}$ by $F_{L}^{\prime}$ and $R$ by $R^{\prime}$. 

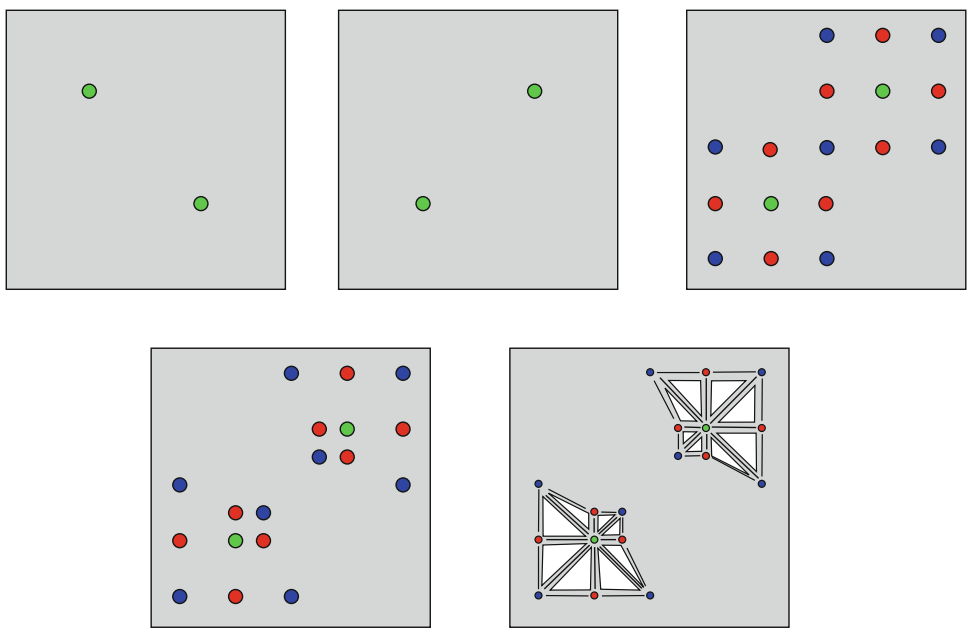

Fig. 4. Computation of $P_{S}(\bar{I})$ : In the raster scan order, we start from $I$, we deduce $\bar{I}$, we compute $F_{J}^{\prime}$ encoding the cells of $Q(\bar{I})$, we construct the set $F_{L}^{\prime}$ and finally obtain the simplicial complex $P_{S}(\bar{I})$ built on the set of vertices $F_{L}^{\prime}$.

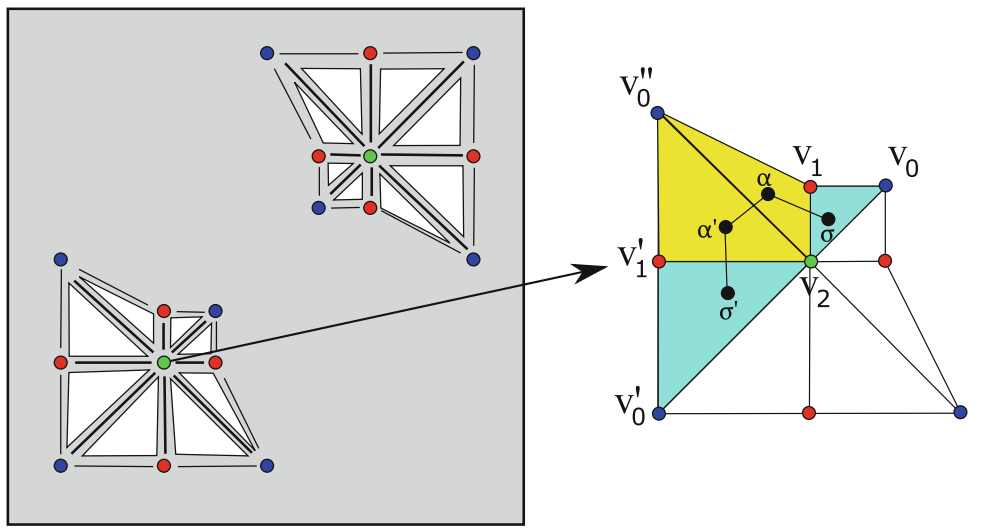

Fig. 5. How to compute the path joining two cells, $\sigma:=\left\langle v_{0}, v_{1}, v_{2}\right\rangle$ and $\sigma^{\prime}:=$ $\left\langle v_{0}^{\prime}, v_{1}^{\prime}, v_{2}\right\rangle$, both incident to $\left\langle v_{2}\right\rangle$. Since we are in the case $i \neq i^{\prime}$, we compute two new cells $\alpha$ and $\alpha^{\prime}$ which "join" $\sigma$ and $\sigma^{\prime}$ : the final path joining $\sigma$ and $\sigma^{\prime}$ is $\pi=\left\langle\sigma, \alpha, \alpha^{\prime}, \sigma^{\prime}\right\rangle$.

Theorem 2. $P_{S}(\bar{I})$ is $w W C$.

Proof. The proof is the same as the one provided for Theorem 44 in [3] where we proved that for any 0 -cell $\mu$ in $P_{S}(I)$, the set of $n$-cells incident to $\mu$, denoted by $\mathcal{A}_{P_{S}(I)}^{(n)}(\mu)$, is face-connected. The same happens for any 0-cell $\mu^{\prime}$ in $P_{S}(\bar{I})$. See an example in Fig. 5. 
Theorem 3. The following properties hold:

(a) $\left|P_{S}(I)\right| \cup\left|P_{S}(\bar{I})\right|=\mathbb{R}^{n}$.

(b) $P_{S}(I) \cap P_{S}(\bar{I})=\partial P_{S}(I)=\partial P_{S}(\bar{I})$.

Proof. (a) $\left|P_{S}(I)\right| \cup\left|P_{S}(\bar{I})\right|=\mathbb{R}^{n}$ :

For a given point $p \in \mathbb{R}^{n}$, consider the closest point $v_{n}(p) \in 4 \mathbb{Z}^{n}=\mathcal{E}_{n}$ under $L^{1}$-norm. Notice that there may be more than one point if $p$ is shared by several $n$-cubes; in that case, take any of them. Then $p$ lies inside the 4 -size $n$-cube $c_{v_{n}(p)}$ centered at $v_{n}(p)$.

- If $v_{n}(p) \notin R \cup R^{\prime}$, then $p$ lies inside a simplicial subdivision of the $n$-cube encoded by $v_{n}(p)$ (that is, there exist $v_{0}, \ldots, v_{n-1}$, with $v_{k} \in \mathcal{C}_{k}$ for all $k \in \llbracket 0, n-1 \rrbracket$ or $v_{k} \in \mathcal{C}_{k}^{\prime}$ for all $k \in \llbracket 0, n-1 \rrbracket$, such that $p$ lies inside $\sigma=\left\langle v_{0}, \ldots, v_{n-1}, v_{n}(p)\right\rangle$ for $\sigma \in P_{S}(I)$ or $\left.\sigma \in P_{S}(\bar{I})\right)$.

- Else, if $v_{n}(p) \in R$, then $\left|c_{v_{n}(p)}\right|$ is a subspace of $|X|$ for

$$
X=\bigcup_{q \in \mathcal{C}_{n} \cap \mathcal{D}_{F_{J}}^{+}\left(v_{n}(p)\right)} q * K_{\mathcal{D}_{F_{L}}}(q) .
$$

Then $p$ lies in $\left|q * K_{\mathcal{D}_{F_{J}}}(q)\right|$ for some $q \in \mathcal{C}_{n} \cap \mathcal{D}_{F_{J}}^{+}\left(v_{n}(p)\right)$.

- Else, $v_{n}(p) \in R^{\prime} \backslash R$. Then $\left|c_{v_{n}(p)}\right|$ is a subspace of $\left|X^{\prime}\right|$ for

$$
X^{\prime}=\bigcup_{q \in \mathcal{C}_{n} \cap \mathcal{D}_{F_{J}^{\prime}}^{+}\left(v_{n}(p)\right)} q * K_{\mathcal{D}_{F_{L}}}(q) \cup \bigcup_{q^{\prime} \in \mathcal{C}^{\prime}{ }_{n} \cap \mathcal{D}_{F_{J}^{\prime}}^{+}\left(v_{n}(p)\right)} q^{\prime} * K_{\mathcal{D}_{F_{L}^{\prime}}}\left(q^{\prime}\right) .
$$

Then $p$ lies in $\left|q * K_{\mathcal{D}_{F_{L}}}(q)\right|$ for some $q \in \mathcal{C}_{n} \cap \mathcal{D}_{F_{J}^{\prime}}^{+}\left(v_{n}(p)\right)$ or in $\mid q^{\prime} *$ $K_{\mathcal{D}_{F_{L}^{\prime}}}\left(q^{\prime}\right) \mid$ for some $q^{\prime} \in \mathcal{C}^{\prime}{ }_{n} \cap \mathcal{D}_{F_{J}^{\prime}}^{+}\left(v_{n}(p)\right)$.

Therefore, $p$ lies in $\left|P_{S}(I)\right|$ or in $\left|P_{S}(\bar{I})\right|$.

(b) $P_{S}(I) \cap P_{S}(\bar{I})=\partial P_{S}(I)=\partial P_{S}(\bar{I})$ :

Let $\sigma$ be a simplex in $\partial P_{S}(I)$. Let us prove that $\sigma \in P_{S}(\bar{I})$. By definition, $\sigma$ belongs to an $(n-1)$-cell $\mu$ of $P_{S}(I)$ that is face of exactly one $n$-cell $\mu^{\prime}=\left\langle v_{0}, \ldots, v_{n}\right\rangle$ of $P_{S}(I)$. We have: $v_{n} \in \mathcal{C}_{n}=\left(\mathcal{E}_{n} \cap F_{L}\right) \cup R$ by construction and $\mu=\left\langle v_{0}, \ldots, v_{n-1}\right\rangle$, with $v_{n-1} \in \mathcal{C}_{n-1}$, otherwise $\mu \notin \partial P_{S}(I)$.

When $v_{n} \in \mathcal{E}_{n} \cap F_{L}$, then $v_{n}$ is the barycenter of a 4 -size $n$-cube of $P_{S}(I)$ and there exists $v_{n}^{\prime} \in \mathcal{E}_{n} \backslash F_{L}$ that is the barycenter of a 4 -size $n$-cube of $P_{S}(\bar{I})$ such that $\left\langle v_{0}, \ldots, v_{n-1}, v_{n}^{\prime}\right\rangle$ is an $n$-cell of $P_{S}(\bar{I})$.

When $v_{n} \in R$ then $v_{n-1} \in S\left(v_{n}\right)$. Since $\mu \in \partial P_{S}(I)$ then $v_{n-1} \in S^{\prime}\left(v_{n}\right) \cap$ $\mathcal{C}_{n-1}^{\prime}$. Therefore, for $\lambda_{j} \in\{0, \pm 1\}$,

$$
v_{n-1}=v_{n}+\sum_{j \in 2_{4}\left(v_{n}\right)} \lambda_{j} e^{j} \text { and then } v_{n}^{\prime}=v_{n}+\sum_{j \in 2_{4}\left(v_{n}\right)} 2 \lambda_{j} e^{j} \in R^{\prime} \backslash R .
$$

Then $\mu$ is an $(n-1)$-face of $\left\langle v_{0}, \ldots, v_{n-1}, v_{n}^{\prime}\right\rangle \in P_{S}(\bar{I})$, so $\mu \in P_{S}(\bar{I})$ and, hence $\sigma \in P_{S}(\bar{I})$.

The proof for $\partial P_{S}(\bar{I})$ is analogous.

If $\sigma \in P_{S}(I) \cap P_{S}(\bar{I})$, then all the vertices of $\sigma$ lie necessarily in $\partial P_{S}(I)=$ $\partial P_{S}(\bar{I})$. 


\section{Conclusions}

In this paper we provide a method to locally repair the cubical complex canonically associated to the complement of a given $\mathrm{nD}$ picture $I$ obtaining a simplicial complex $P_{S}(\bar{I})$. We prove that $P_{S}(\bar{I})$ decomposes the complement space of the continuous analog of $P_{S}(I)$ and is weakly well-composed. As future works, we plan to study the combinatorial structure of $\partial P_{S}(I)=\partial P_{S}(\bar{I})$ and prove that it is a combinatorial $(n-1)$-manifold.

Acknowledgments. This research has been partially supported by MINECO, FEDER/UE under grant MTM2015-67072-P and Instituto de Matematicas de la Universidad de Sevilla (IMUS).

\section{References}

1. Bhunre, P.K., Bhowmick, P., Mukherjee, J.: On efficient computation of intersimplex Chebyshev distance for voxelization of 2-manifold surface. Inf. Sci. (2018)

2. Boutry, N., Géraud, T., Najman, L.: A tutorial on well-composedness. J. Math. Imaging Vis. 60(3), 443-478 (2018)

3. Boutry, N., Gonzalez-Diaz, R., Jimenez, M.J.: Weakly well-composed cell complexes over nD pictures. Inf. Sci. (2018)

4. Boutry, N., Géraud, T., Najman, L.: How to make $n \mathrm{D}$ functions digitally wellcomposed in a self-dual way. In: Benediktsson, J.A., Chanussot, J., Najman, L., Talbot, H. (eds.) ISMM 2015. LNCS, vol. 9082, pp. 561-572. Springer, Cham (2015). https://doi.org/10.1007/978-3-319-18720-4_47

5. Comic, L., Nagy, B.: A topological 4-coordinate system for the face centered cubic grid. Pattern Recognit. Lett. 83, 67-74 (2016)

6. C̃omić, L., Magillo, P.: Repairing 3D binary images using the BCC grid with a 4-valued combinatorial coordinate system. Inf. Sci. (2018)

7. Dey, T.K., Li, K.: Persistence-based handle and tunnel loops computation revisited for speed up. Comput. Graph. 33(3), 351-358 (2009)

8. Dey, T.K., Li, K., Sun, J.: On computing handle and tunnel loops. In: IEEE International Conference on Cyberworlds, 357-366. IEEE (2007)

9. Floater, M.S., Hormann, K.: Surface parameterization: a tutorial and survey. In: Dodgson, N.A., Floater, M.S., Sabin, M.A. (eds.) Advances in Multiresolution for Geometric Modelling, pp. 157-186. Springer, Heidelberg (2005). https://doi.org/ 10.1007/3-540-26808-1_9

10. Gonzalez-Diaz, R., Jimenez, M.-J., Medrano, B.: Well-composed cell complexes. In: Debled-Rennesson, I., Domenjoud, E., Kerautret, B., Even, P. (eds.) DGCI 2011. LNCS, vol. 6607, pp. 153-162. Springer, Heidelberg (2011). https://doi.org/ 10.1007/978-3-642-19867-0_13

11. Gonzalez-Diaz, R., Jimenez, M.J., Medrano, B.: 3D well-composed polyhedral complexes. Discrete Appl. Math. 183, 59-77 (2015)

12. Gonzalez-Diaz, R., Jimenez, M.-J., Medrano, B.: Encoding specific 3D polyhedral complexes using 3D binary images. In: Normand, N., Guédon, J., Autrusseau, F. (eds.) DGCI 2016. LNCS, vol. 9647, pp. 268-281. Springer, Cham (2016). https:// doi.org/10.1007/978-3-319-32360-2_21 
13. Gonzalez-Diaz, R., Jimenez, M.J., Medrano, B.: Efficiently storing well-composed polyhedral complexes computed over 3D binary images. J. Math. Imaging Vis. 59(1), 106-122 (2017)

14. Lachaud, J.-O., Thibert, B.: Properties of Gauss digitized shapes and digital surface integration. J. Math. Imaging Vis. 54(2), 162-180 (2016)

15. Massey, W.S.: A Basic Course in Algebraic Topology. Springer, New York (1991) 